\title{
Langue bretonne, nation française, République jacobine et perspective européenne
}

Francis Favereau

\section{OpenEdition}

1 Journals

Édition électronique

URL : http://journals.openedition.org/ries/4231

DOI : 10.4000/ries.4231

ISSN : 2261-4265

Éditeur

Centre international d'études pédagogiques

Édition imprimée

Date de publication : 1 septembre 1994

Pagination : 75-84

ISSN : 1254-4590

\section{Référence électronique}

Francis Favereau, «Langue bretonne, nation française, République jacobine et perspective européenne », Revue internationale d'éducation de Sèvres [En ligne], 03 | 1994, mis en ligne le 17 avril 2015, consulté le 02 mai 2019. URL : http://journals.openedition.org/ries/4231 ; DOI : 10.4000/ ries. 4231

Ce document a été généré automatiquement le 2 mai 2019.

(c) Tous droits réservés 


\title{
Langue bretonne, nation française, République jacobine et perspective européenne
}

\author{
Francis Favereau
}

1 Deux faits politiques majeurs tendent à faire évoluer la problématique des langues minoritaires de France en cette fin du XXe siècle : l'émergence de l'Europe d'une part et, de l'autre, une certaine régionalisation des pouvoirs (issue de la loi Defferre de 1982). Ces évolutions, qui ne sont d'ailleurs peut-être que les deux faces d'un même mouvement, d'essence plus ou moins fédérale, voire fédéraliste, ont tendance à prendre en tenaille une logique séculaire : celle d'une République «une et indivisible » jusque sur le plan linguistique, notamment depuis la III ${ }^{e}$ République (lois Ferry), et, plus avant dans le temps, selon les principes révolutionnaires de la nouvelle nation française, laquelle devait sonner le glas des divers idiomes ou parlers assimilés à l'Ancien Régime. Après avoir examiné brièvement ce contexte historique ${ }^{1}$, nous tenterons d'apprécier en quoi l'actuelle construction de l'Europe a pu peser sur l'évolution de la politique linguistique de la France.

\section{Les origines du jacobinisme linguistique}

2 Certains rappels historiques s'imposent car, apparemment, l'arsenal législatif et réglementaire hérité de la Révolution n'est toujours pas caduc, puisqu'il est parfois mis en avant par les autorités (académiques ou ministérielles), dès qu'il s'agit de reconnaissance officielle du bilinguisme. Ainsi, en 1981, les autorités finistériennes opposèrent-elles à des parents, désireux d'ouvrir une école Diwan à Pont-L'Abbé, le décret du 30 vendémiaire an II (21/X/1793), qui stipulait (à propos des élèves des «maisons communes d'éducation ») : «Ils apprennent à parler, lire, écrire la langue française » (exclusivement donc), ainsi que le décret du 7 brumaire an II : «Dans toutes les parties de la République, l'instruction ne se fait qu'en langue française ${ }^{2}$.» 
D'autres exemples récents pourraient être cités ici, comme les propos tenus par des recteurs d'académie, ou des ministres d'État, pour s'opposer de la même façon à la signature de la Charte européenne des langues régionales ou minoritaires.

Certes, l'Ancien Régime n'avait pas été en reste en matière de politique centralisatrice. Et les mêmes responsables politiques s'empressent généralement de citer l'édit de VillersCotterêts (pris par François I Ir en 1539, peu de temps donc après le rattachement de la Bretagne à la couronne de France, même si celui-ci s'opposait d'abord à l'usage administratif du latin). Comme si un édit de l'Ancien Régime s'imposait à nous, à la différence des autres "droits et privilèges" supprimés allègrement dans la nuit du 4 août! Il faut bien admettre que ce mépris envers les «idiomes » fit l'objet d'un large consensus chez les écrivains français des siècles classiques qui, tous ou presque (La Fontaine, $\mathrm{M}^{\mathrm{me}}$ de Sévigné, Voltaire, etc.), eurent leur couplet cinglant sur le bas-breton. C'est que le breton armoricain bénéficiait, depuis le XIII ${ }^{\mathrm{e}}$ siècle, grâce à un accord entre le Duché et la Papauté, d'un statut de langue officielle (la « règle d'idiome»), qui imposait son usage dans toutes les relations entre l'Église et le peuple de Basse-Bretagne, y compris donc l'enseignement, totalement contrôlé par les clercs et les recteurs de paroisse. Aussi paraitt-il difficile de parler d'ostracisme linguistique jusqu'à la fin du XVIII ${ }^{\mathrm{e}}$ siècle, même si la tendance naturelle allait dans le sens d'une certaine francisation (échanges externes, urbanisation et développement du pouvoir d'État, justice et fiscalité surtout, mais aussi ordres religieux contemplatifs). Toutefois, le nombre de locuteurs continuera d'augmenter, essentiellement du fait de la démographie, jusqu'au «million de bretonnants » du tournant du siècle, pour atteindre son apogée entre 1900 et 1914 avec quelque 1,4 million (estimé, faute de tout recensement).

5 Ce n'est pas que la Révolution française ait provoqué une rupture brutale dans un premier temps. Elle est même bien accueillie, de prime abord, en Bretagne (avant que la conscription et la Constitution civile du clergé ne débouchent sur certaines résistances d'une population que l'on estime aujourd'hui avoir été largement "réfractaire ", et ne provoquent la Chouannerie dans une petite moitié du territoire breton - sur d'autres thèmes, il est vrai).

6 La politique linguistique de l'Assemblée constituante ne diffère guère de celle de l'Ancien Régime finissant. À l'instar des États généraux, elle engage une dynamique de traduction (en «bas-breton») des divers textes de loi $^{3}(17 / \mathrm{I} / 1790)$, augmente les salaires des enseignants bilingues $(14 / \mathrm{V} / 1790)$ et assure le paiement de commissions pour la traduction de ses décrets (6/XI/1792 - « en idiomes vulgaires bas-breton, basque, etc. »). La raison en est qu'en Basse-Bretagne, comme au Pays basque, le seul médiateur entre le nouveau pouvoir central et le peuple paysan est le recteur de paroisse, qui informe publiquement ses ouailles lors du prône dominical. La traduction officielle assure donc une ombre de contre-pouvoir, avec l'espoir parfois exprimé qu'une paysannerie désormais éclairée (dans sa langue donc) se détachera mécaniquement de ses prêtres réactionnaires...

7 La Convention marquera un changement brutal dans cette politique, somme toute plutôt positive. Le premier texte qui fait autorité est le rapport Barrère (Rapport du Comité de Salut public sur les idiomes, du 8 pluviôse an II - 27/I/1794). Il a une forte coloration idéologique :

Nous avons révolutionné le gouvernement, les lois, les usages, les mœurs, les costumes, le commerce et la pensée même; révolutionnons donc aussi la langue, qui est leur instrument journalier. 
Puis :

Le fédéralisme et la superstition parlent bas-breton : l'émigration et la haine de la République parlent allemand; la contre-révolution parle l'italien, et le fanatisme parle le basque. Cassons ces instruments de dommage et d'erreur.

9 Enfin :

Citoyens, la langue d'un peuple libre doit être une et la même pour tous.

Le second texte essentiel est le rapport Grégoire, abbé fort célébré par ailleurs, intitulé Rapport sur la nécessité et les moyens d'anéantir les patois et d'universaliser l'usage de la langue française, du 28 prairial an II - 16/VI/1794 - par Grégoire ( sic). Lui non plus ne s'embarrasse guère de nuances :

Nous n'avons plus de provinces, et nous avons encore environ trente patois qui en rappellent les noms... le bas-breton, le normand, le picard, le rouchi, le wallon... Au nombre des patois, on doit encore placer l'italien de la Corse, des Alpes-Maritimes, et l'allemand des Haut et Bas-Rhin, parce que ces deux idiomes y sont très dégénérés. Enfin les nègres de nos colonies, dont vous avez fait des hommes, ont une espèce d'idiome pauvre comme celui des Hottentots...

D'où cette volonté :

Cette entreprise, qui ne fut pleinement exécutée chez aucun peuple, est digne du peuple français, qui centralise toutes les branches de l'organisation sociale et qui doit être jaloux de consacrer au plus tôt, dans une République une et indivisible, l'usage unique et invariable de la langue de la liberté.

Cette idéologie de rupture révolutionnaire et de purification linguistique, en somme, entra en application peu après par la loi du 27 brumaire an II, sur l'instruction publique, qui stipule (ch. IV, art. 2) :

L'enseignement sera fait en langue française. L'idiome du pays ne pourra être employé que comme moyen auxiliaire.

Force est de constater que cette politique offensive n'entrera guère dans les faits. La création d'un nouveau corps d'instituteurs dits « de langue française » (afin de détruire le breton, « idiome esclave incompatible avec l'unité de la République ») est un échec total ${ }^{4}$. Certes, cette politique répondait d'abord au syndrome d'une révolution assiégée par la vieille Europe, précisément, avec la peur d'une contagion aux frontières, peuplées de minorités allogènes (allemandes, flamandes, italiennes, basques...). Or la Convention n'aura été, sur ce plan, qu'une brève parenthèse extrémiste (et même totalitaire, tout au moins sur ce plan), dans un processus qui s'inscrit dans la longue durée. Cependant ces rudes préceptes resteront, et restent encore invoqués, à l'encontre de tous les défenseurs des langues minoritaires, d'autant que la Révolution française fait l'objet d'un culte régulier et de célébrations qui n'encouragent guère le dialogue, même républicain, sur le sujet!

Le XIX ${ }^{\mathrm{e}}$ siècle sera, dans ce domaine, impuissant à mettre en œuvre des principes jamais reniés, et rarement contredits. Ainsi, en 1831, la circulaire de Montalivet, ministre de l'instruction, qui ouvrait la porte aux langues comme le breton, rencontra un accueil mitigé (soutien enthousiaste du préfet du Morbihan, mais opposition farouche du préfet du Finistère, qui corrobore l'attitude ouvertement colonialiste - sic - d'Auguste Romieu, sous-préfet de Quimperlé, et écrivain à la mode, dans un article de la Revue de Paris, tome XXX, 1831). La loi Guizot (1833), elle-même, n'est guère appliquée à la lettre, l'instruction demeurant en Bretagne l'apanage de l'Église, et le breton étant resté traditionnellement la langue du catéchisme, de l'instruction religieuse, voire de la 
lecture. Les chiffres sont parlants : selon notre collègue historien Michel Lagrée, seul un quart des écoles finistériennes n'utilisait pas le breton, et la proportion est à peu près la même dans la partie bretonnante du Morbihan et des Côtes-du-Nord d'alors (d'après une enquête de 1863) ${ }^{5}$. C'est aussi l'impression laissée par les témoignages et les études ${ }^{6}$.

Tout va changer avec l'instauration de la III ${ }^{\mathrm{e}}$ République, qui va parvenir à mettre en œuvre les "grands principes » de la Révolution avortée, surtout avec l'application des lois Ferry sur l'école publique, qui marqueront des générations de bretonnants, et d'autres, jusqu'à nos jours.

\section{L'exclusion par la République}

La Révolution, nous l'avons vu, avait imposé dans les esprits l'équation « école = français ». On retrouve donc peu ou prou l'analyse de Barère, passée de nouveau dans les faits : aux Chouans, puis aux « chouans » (en politique) et à leurs curés, leur basbreton; à la Nation, à la République, au Progrès, la langue française et ses valeurs universelles. Telle est l'idéologie simpliste, mais terriblement efficace, qui va s'imposer à la fin du XIX siècle et perdurer par la suite. Et cela d'autant plus facilement que la vie politique, au travers de la question scolaire notamment, verra se perpétuer une logique d'affrontement manichéen, héritée de la Révolution française, entre cléricalisme militant et laïcisme républicain. Gilbert Nicolas vient d'analyser dans un livre important cette politique de formation des instituteurs dans la première école normale primaire de Rennes entre 1831 et 1850 : ils se devaient d'être les relais efficaces d'un État qui entendait déjà, dit-il, «franciser le langage » et « moudre les différences »

列 une stratégie très cohérente visant à l'exclusion totale du breton comme des autres « idiomes ». L'article $14 \mathrm{du}$ règlement des écoles primaires est très net à cet égard : «Le français sera le seul en usage ». Inspecteurs d'académie, ou inspecteurs primaires, tous donneront, en aval, des directives on ne peut plus fermes aux nouveaux « hussards noirs de la République " issus des écoles normales départementales nouvellement créées (et succédant à celle, unique, de Rennes, au départ). Ces rapports ont été étudiés et publiés récemment par Claude Le Du dans son Histoire d'un interdit : le breton à l'école 8 .

Dans cette stratégie très au point, un symbole puissant apparaît (ou réapparaît) : le «symbole» précisément. Il s'agit d'un objet dérisoire de bois, ou bien encore d'un caillou... dit parfois "vache» ou "sabot», et dont le souvenir est toujours vivant. L'interdiction de parler breton en classe, que l'on justifiait par la nécessité d'un «bain linguistique ", à un moment où l'école était le seul centre francophone en zone rurale, se voit ainsi prolongée par un interdit qui frappe la langue jusque dans la cour de récréation, et encourage la délation'.

19 La réaction de la population est bien difficile à sonder un siècle plus tard, même si l'on admet qu'elle adhéra à l'idéologie du "progrès » de la République sociale, tout en conservant sa langue, dans un premier temps, peut-être plus grâce à sa force d'inertie, que par choix délibéré ${ }^{10}$. Par contre, les réactions politiques, plus tranchées, nous ramènent au vif du sujet. La tendance hégémonique, celle du bloc rural, est bien illustrée par Le courrier du Finistère (journal influent, de la tendance de l'évêché quimpérois toutpuissant, d'abord légitimiste puis conservateur). Son rédacteur comprend cette exclusion du breton de l'école comme une menace pour l'école catholique elle-même, par ricochet : 
Aucun risque pour autant que les enfants ne soient dépossédés du breton: ils l'entendront et le parleront à la maison, et ce sera suffisant pour la majorité d'entre eux. $(24 / 11 / 1908)$ futurs cultivateurs, et encore moins de leurs épouses. Quant aux demandes formulées par des universitaires comme Joseph Loth (chargé de cours de « celtique » depuis la fin du XIX ${ }^{e}$ siècle et dont la chaire fut agréée en 1903, à condition qu'elle fût financée par les cinq Conseils généraux!), ou Anatole Le Braz, pourtant "bon républicain », elles se heurtèrent toujours au même refus obstiné et poli des ministres, notamment au moment du combisme, qui reste comme une caricature absolue du radicalisme de la III ${ }^{e}$ République au prétexte que toute ouverture en la matière serait une brèche où s'engouffrerait le « séparatisme » breton (pourtant ultra-minoritaire). Il n'est pas jusqu'au socialiste irlandais James Connolly qui ne s'en offusque dans sa correspondance avec ses homologues français d'avant 1914 ! Cette obstination se comprend d'autant mieux que cette III République est, depuis ses origines, avec la figure du Lorrain Jules Ferry notamment, comme avec la caution d'Ernest Renan (celtisant entre autres), ardemment colonialiste, prétendant imposer ses Lumières par sa culture, et d'abord par sa langue, en Afrique (Algérie particulièrement) comme en Extrême-Orient !

D'autres tendances se font jour, et une autre orientation eût été possible, comme en témoigne l'éphémère revue Brug, d'esprit libertaire, qui, avant 1914, prend le contrepied des idéologies dominantes, tant républicaine que cléricale, dans une émouvante défense du breton à l'école, pour arracher le peuple paysan de Bretagne au cléricalisme, au profit d'un internationalisme généreux et pacifique, selon les théories de son directeur Émile Masson, "saint anarchiste ", qui fait exception dans ce milieu laïc "rouge et noir », et sauve du moins l'honneur. Dans l'entre-deux guerres, alors que l'école de la République continue sur sa lancée, des mouvements revendicatifs vont s'affirmer, et se référer souvent à l'Europe ou aux colonies, selon leurs préférences idéologiques. Ar Falz, mouvement laïque, s'appuie sur le modèle des républiques soviétiques de l'URSS, « phare des peuples » pour tant de progressistes (cf. Ar Falz n 1, janvier 1933), et sur la solidarité avec les langues indigènes. Force est pourtant de constater que son action demeurera surtout symbolique et ne portera ses fruits qu'après-guerre. Le mouvement catholique BABES (Breuriez ar brezoneg er skoliou), comme les revues littéraires nationalistes (Gwalarn...), prendront d'abord exemple sur les minorités d'Europe catholiques (l'Irlande surtout, ou la "Bohème ", voire la Pologne, et bientôt le Pays basque), avant de chercher des modèles dans l'Europe du nord (au Danemark pour ses "écoles du peuple ", ou en Norvège). Or, pétitions et vœux, même repris par un large mouvement d'élus, plus neutre, ABES (Ar brezoneg er skol), rien n'y fera! En 1936, le refus opposé par le ministre de l'instruction publique à une demande du marquis de Kerouartz est tout aussi catégorique que du temps d'Anatole de Monzie, qui déclarait en 1923 : «Pour l'unité linguistique de la France, la langue bretonne doit disparaître ». Du côté des évêchés, la hiérarchie se fera toutefois plus réceptive aux propositions de BABES, comme le prouvent les instructions de $\mathrm{M}^{\mathrm{gr}}$ Duparc (évêque de Quimper et Léon, cf. Feiz ha Breiz, 1929, n 9, ou 1936, n 1, par exemple), et elle mettra en place un certain enseignement facultatif de la langue et de l'histoire de Bretagne, particulièrement en Cornouaille intérieure (sous l'impulsion d'un groupe de militants bretons), mais aussi en Léon, en Trégor et en Vannetais. Ceci débouchera sur l'arrêté Carcopino de 1941, autorisant l'ouverture de cours facultatifs dans les écoles primaires et la création d'épreuves au certificat, non pas tant dans le cadre de la «révolution nationale » du maréchal Pétain, ou de "l'ordre nouveau européen », 
que sous la pression de ces divers mouvements bretons, qui auront désormais le champ libre. D'autant que la III ${ }^{e}$ République, dès lors, a vécu !

La reconnaissance de la langue bretonne avait été, entre les deux guerres, comme avant 1914, la première revendication de tous ces mouvements bretons, qu'ils soient culturels, régionalistes ou autonomistes, modérés ou tentés de verser dans l'extrémisme, séparatiste ou bien fasciste. Autant dire donc que la problématique d'une langue minoritaire comme le breton, au cœur d'une « question bretonne » plus large, s'inscrivait pleinement dans un cadre européen, lequel était alors désastreux pour elle. Seul «l'État libre » d'Irlande offrait en effet une vague alternative positive, et encore sa révolution a$\mathrm{t}$-elle eu un attrait des plus ambigus sur la mouvance politique bretonne ${ }^{11}$.

\section{Les contre-modèles européens}

C'est, paradoxalement, la Libération qui aidera à sortir de cette impasse. Si une première proposition de loi, déposée par les députés communistes bretons (Hamon et Hervé, récemment disparus), n'aboutit pas, la SFIO, devenue parti-charnière, fait voter la loi Deixonne (1950-1951), qui permet l'introduction des langues régionales au lycée. Première ouverture timide, imposée par les députés du Midi (qu'on dirait aujourd'hui occitans), elle ne dut d'être appliquée (par la signature des décrets, selon le témoignage du militant laïc breton Keravel) qu'à la pression du nouveau président du Conseil, René Pleven, ancien de Londres, et bientôt notable centriste tout-puissant en Côtes-du-Nord, européen convaincu, comme l'on sait. Si la logique de la loi Deixonne obéissait surtout à un débat franco-français et répondait à la pression des milieux résistants et progressistes, entre autres, l'environnement européen était, là encore, bien présent: début de la construction européenne, CED comprise, ainsi que les prémices de la décolonisation.

Il faut attendre 1971 pour trouver une nouvelle avancée, avec le décret Guichard, prévoyant les cours rémunérés en lycée. Et encore s'agit-il là d'une « conquête » typique des années post-68, où le « pouvoir fort » des gaullistes alors à l'œuvre cédait déjà, comme à regret, à la pression des marches et des manifestations de rues, appuyées par les syndicats et les partis de gauche. Georges Pompidou ne déclarait-il pas, en effet, en 1973 encore, qu'«il n'y a pas de place pour les langues régionales dans une France destinée à marquer l'Europe de son sceau »!

Le septennat de Giscard d'Estaing marque, sans doute, comme dans d'autres domaines, la vraie rupture - quoique molle - avec ce jacobinisme bonapartiste hérité du XIX ${ }^{e}$ siècle, en passant par la III République, et illustré encore naguère par l'intransigeance verbale d'un Michel Debré12 et de ses successeurs, encore que de Gaulle lui-même ait toujours tenu deux discours opposés sur ces questions. La "Charte culturelle de Bretagne », signée en $1977^{13}$, entre l'État et les collectivités locales en plein développement - la région et les Conseils généraux - prévoyait, parmi d'autres mesures, un réel enseignement en collège, comme option, puis comme LV2 (en 1979), et des aides substantielles aux associations culturelles ainsi qu'à l'édition.

La question linguistique entra alors dans le débat politique, le PS épousant dans l'opposition les thèses d'un mouvement breton désormais nettement lévogyre. Et, de fait, les années Mitterrand seront marquées par diverses avancées notables, non sans luttes préalables et tensions multiples, car les mesures prévues se heurteront constamment aux résistances ou aux valses-hésitations ministérielles: Alain Savary a été le pionnier de 
l'ouverture, en comparaison du très jacobin Jean-Pierre Chevènement, et même de Lionel Jospin, théoriquement favorable, avant que Jack Lang ne fasse des déclarations très «prometteuses »... mais sur le tard! Sans parler du ministère Chirac, peu enclin à des avancées malgré un préjugé favorable du ministre en titre, René Monory. Le béarnais F. Bayrou a, en 1993, élargi l'option « langue régionale », mais il semble aujourd'hui en panne sur ce plan, malgré des déclarations très nettes:

C'est fini le temps de la honte. Commence, quand bien même il serait tard, quand bien même il serait difficile, le temps de la fierté. Et la fierté n'est pas seulement une déclaration [...] C'est aussi une politique [...] Oui, nous allons retrouver la langue à l'école [...] (Discours au Parlement de Navarre à Pau, le 25 octobre 1993).

Quant aux mesures concrètes de ces années 1980, ce furent, dans l'ordre, la licence de breton créée en 1981, le CAPES en 1984-1985 (annoncé par François Mitterrand dans un meeting à Rennes !), puis le DEUG en 1989, ainsi que la création et le développement de filières bilingues ces dernières années.

Il faut bien sûr accorder une mention spéciale aux écoles Diwan, créées en 1977, sur le modèle des premières ikastolak associatives et autogérées en langue basque. Ces écoles, créées hors du système, ont sûrement servi d'aiguillon à l'enseignement public, puis plus récemment à l'enseignement catholique qui vient lui aussi de créer plusieurs classes bilingues, avec un appui financier important de Conseils généraux (Finistère, puis Morbihan et Côtes-d'Armor). Quelque 2000 élèves sont ainsi scolarisés en bilingue, alors qu'environ 21000 (chiffres officiels, cités par Le Télégramme, 1994) bénéficient d'une certaine initiation à la langue bretonne ${ }^{14}$.

29 Il faut donc bien admettre que ce n'est qu'après un bon siècle d'un dur ostracisme, particulièrement durant toute la période cruciale couverte par la III ${ }^{e}$ République, et à la veille d'une mort annoncée longtemps à l'avance, que le breton a enfin acquis droit de cité en France ! Légèrement plus tard - faut-il le répéter ? - que le basque en Espagne, par exemple, peu après la chute du franquisme ; bien longtemps après le gallois ou le gaélique dans une Grande-Bretagne pourtant réputée terriblement conservatrice, elle qui admit le gaélique à l'école en Irlande dès la fin du XIXe siècle. Le terme de "réparation historique ", avancé par Henri Giordan (qui fut conseiller de F. Mitterrand sur la question), et parfois contesté, n'aurait donc rien eu d'exagéré. Le paradoxe veut que l'opinion publique, selon un récent sondage du Haut conseil national des langues régionales de France (du $1^{\mathrm{er}}$ avril 1994), est à plus des trois-quarts (de $75 \%$ à $90 \%$ selon les questions) globalement favorable aux dites langues régionales. N'est-ce pas ce qu'on appelle, dans la France des « hommes d'État», gouverner contre les sondages?

La construction européenne, ainsi donc, a joué un rôle primordial. Sur un plan géopolitique, tout d'abord, elle a permis de liquider le contentieux franco-allemand et donc la question de l'Alsace-Lorraine, si importante jadis au regard de 1'«unité nationale » qu'elle mettait en cause. Elle a contribué ensuite à donner du poids aux régions, à l'instar des Länder, et aux autres collectivités locales en la matière (enseignement bilingue, édition, signalisation bilingue...). Enfin elle a introduit, avec la Grande-Bretagne, un contre-modèle culturel et scolaire, basé sur la déconcentration et le pragmatisme, qui fait que les conservateurs britanniques ont pu engager, sous la pression certes, des politiques hardies en faveur du gallois, puis du gaélique d'Écosse (bilinguisme généralisé, scolaire et administratif, chaîne de télévision, aides à l'édition et à la création, etc.). Cette politique «régionale » à l'anglaise est d'ailleurs plus hardie que celle de la République d'Irlande en faveur de sa langue officielle, l'irlandais (très proche dudit 
gaélique d'Écosse !). De même, est-il difficile d'ignorer l'écho qu'a eu l'exemple espagnol en deçà des Pyrénées, alors qu'au-delà, Pays basque et Catalogne ont pu, grâce à une large autonomie, développer des politiques importantes de "normalisation linguistique ", pratiquement dès la sortie du franquisme, et se doter de grands médias (journaux, radios, télévisions...) dans leur langue, ainsi que de systèmes scolaires propres. Ce n'est pas un hasard si les liens entre Bretagne et Catalogne ou Pays basque sont si importants, et si les jumelages entre communes bretonnes et galloises ou irlandaises si nombreux. Et la Belgique, ou l'Italie, entre autres, offrent depuis longtemps divers autres exemples de respect de leurs minorités linguistiques.

31 L'Europe a également offert de nouvelles perspectives, tant associatives, avec des groupes de pression organisés (tels le European Bureau for lesser used languages, où le breton a son mot à dire, et donc plus aisément à Bruxelles qu'à Paris), qu'institutionnelles, avec la Charte européenne des langues régionales ou minoritaires. Sans compter des programmes européens d'échanges comme Erasmus-Celtic Studies, qui regroupe une vingtaine d'universités, et bien d'autres réseaux (Lingua, Mercator...), et bientôt Socrate. Il est, cependant, navrant de constater que, les mêmes causes produisant les mêmes effets, les gouvernements successifs ont refusé de signer ladite Charte, acceptée dans maints États-nations pourtant, tout comme ils ont évité de discuter une proposition de loi qui s'annonçait intéressante (proposition de loi Dollo, la $42^{\mathrm{e}}$ à être éconduite, depuis les années 1870). Cette analyse ne risque pas d'être démentie par le récent débat sur la loi Toubon (concédant simplement un certain droit à n'être pas niées ou combattues pour nos langues régionales, à la demande en particulier du sénateur alsacien Goetschy) : le texte ne "s'oppose pas à [l'] usage» des langues régionales (selon Le Monde du 16 avril 1994)!

La situation paraît aujourd'hui figée, voire bloquée, au niveau de l'État central. Les déclarations d'intention du ministre F. Bayrou, plus lyriques que notre modeste propos, et c'est très bien ainsi, n'ont apporté que de maigres mesures, et peu de résultats tangibles. Qu'en sera-t-il à l'avenir ? La France devra-t-elle se trouver un peu plus isolée, Grèce et Turquie mises à part, comme elle l'est parfois sur d'autres dossiers, avant d'abandonner une position objectivement à contre-courant de l'évolution européenne, et par conséquent, devenue intenable ? Ou bien se cramponnera-t-elle à des certitudes d'un autre âge, datant des guerres révolutionnaires, puis de la "guerre civile européenne ", jusqu'à ce que le système, scolaire ou politico-médiatique, également centralisés, n'implose sous nos yeux, pour aller vers "l'après-France ", selon le mot du professeur occitan Robert Lafont ? On ne sait. Mais le doute et le désenchantement sont réels, tant la situation semble bloquée et l'horizon tout à fait bouché. La "machine " est, en effet, terriblement indifférente, quand elle ne leur est pas hostile, à la cause des langues minoritaires. Du moins aurons-nous eu la satisfaction d'avoir pu donner ici notre sentiment sur un sujet qui nous occupe ! 


\section{NOTES}

1. Nous en avons proposé une étude plus détaillée dans : Francis Favereau, Bretagne contemporaine - langue, culture, identité, Skol Vreizh, Morlaix, 1993.

2. Daniel Bernard, «La Révolution française et la langue bretonne ", Annales de Bretagne, tome XXVIII, 1912- 1913, p. 310-312.

3. Andreo Merser, 1789 hag ar brezoneg, Brud nevez, 1989.

4. F. Favereau, op. cit., p. 146.

5. Michel Lagrée, Religion et cultures en Bretagne, Fayard, 1992, p. 240.

6. J'en ai cité plusieurs exemples ou extraits dans mon ouvrage mentionné à la note 1 .

7. G. Nicolas, Instituteurs entre politique et religion. La première génération de normaliens en Bretagne au XIX ${ }^{e}$ siècle, Apogée, Rennes, 1993.

8. Klaoda an du, Histoire d'un interdit - le breton à l'école, Hor Yezh, 1992.

9. Pour la période 1912-1960, la grande majorité des témoignages recueillis (hormis sept cas sur cent trente) par Claude Le Du, conseillère pédagogique dans les Côtes-d'Armor, va dans ce sens. Un certain bilinguisme de fait fait exception, toléré plus qu'encouragé, car l'on a plutôt affaire alors à des démarches individuelles, ou plus ou moins militantes, marginales, et dont le laxisme est à l'occasion très vivement critiqué par les autorités académiques.

10. Le lecteur est invité à se reporter à mon étude (op. cit., p. 152-155).

11. Francis Favereau, «Irlande, Irlandais, \& irlandais (gaélique) dans les lettres bretonnes depuis la dernière guerre ", L'Irlande et ses langues, PUR, 1992, p. 43-52.

12. Cf. les articles de Y.B. Piriou dans Les lettres françaises de 1962.

13. Cf. P. Bernard (alors député européen de Lorient), «Les chances culturelles de la Bretagne », 1986, (réédité dans Ar Falz n 57).

14. Le statut d'écoles privées non confessionnelles sous contrat de l'État a été accordé tout récemment aux écoles Diwan par François Bayrou, ministre de l'Éducation nationale (NDLR).

\section{RÉSUMÉS}

À travers l'exemple du breton, la question des langues minoritaires de France apparaît, en cette fin de siècle, évoluer lentement du fait de la régionalisation et de la dynamique européenne. Leurs effets combinés renverseront-ils la tendance historique de l'uniformisation linguistique?

\section{INDEX}

Index géographique : France

Mots-clés : langue bretonne, politique linguistique, Conseil de l'Europe, langue régionale 


\section{AUTEUR}

\section{FRANCIS FAVEREAU}

Université de Haute-Bretagne-Rennes II, directeur du laboratoire Bretagne et Pays celtiques 